\title{
The quasi-adiabatic approximation for coupled thermoelasticity
}

\author{
Aleksey V. Pichugin ${ }^{1}$ \\ Department of Mathematical Sciences, Brunel University, UB8 3PH, UK
}

\begin{abstract}
The equations of coupled thermoelasticity are considered in the case of stationary vibrations. The dimensional and order-of-magnitude analysis of the parameters occurring within these equations prompts the introduction of the new non-dimensionalisation scheme, highlighting the nearly-adiabatic nature of the resulting motions. The departure from the purely adiabatic regime is characterised by a natural small parameter, proportional to the ratio of the mean free path of the thermal phonons to the vibration wavelength. When the governing equations are expanded in terms of the small parameter, one can formulate an equivalent "quasi-adiabatic" system of the equations of ordinary elasticity with frequency-dependent modulae, characterising the thermoelastic dissipation. Unfortunately, this model lacks the degrees of freedom necessary to satisfy boundary condition(s) for the temperature. Thus, we also derive a complementary boundary layer solution and show that to the leading order it is described by thermoelastic equations in the quasi-static approximation. Further simplifications are possible for purely dilatational motions; we illustrate this point by solving a model thermoelastic problem in 1D.
\end{abstract}

Keywords: vibration, thermoelastic dissipation, coupled thermoelasticity, quasi-adiabatic approximation, asymptotic, boundary layer. PACS: $46.25 . \mathrm{Hf}, 46.15 . \mathrm{Ff}, 46.40 .-\mathrm{f}, 46.35 .+\mathrm{z}$.

It is known that in most regularly-sized bodies lowest vibration modes occur at frequencies that lie well below the characteristic frequency $\omega_{*}$, in the neighbourhood of which the thermoelastic response undergoes the transition from predominantly adiabatic $\left(\omega \ll \omega_{*}\right)$ to predominantly isothermal $\left(\omega \gg \omega_{*}\right)$, see [1]. The direct practical consequence of this fact concerns experimental measurements of the elastic modulae; while typical static experiments measure the isothermal values of the elastic constants, typical dynamic experiments measure their adiabatic values. The adiabatic conditions are usually satisfied to such an extent that another major manifestation of the thermoelastic coupling - the thermoelastic dissipation - is often negligible in comparison with other loss factors. Nevertheless, this observation becomes untrue for smaller bodies, so much so, that oscillations of micro- and nano-electromechanical systems (MEMS/NEMS) are appreciably affected by thermoelastic dissipation. In this paper we aim to expose the analytical structure of the equations of thermoelasticity in the adiabatic limit, and to construct simplified asymptotic models capable of describing nearly adiabatic vibrations typical for the practical thermoelastic resonators.

\section{GOVERNING EQUATIONS}

The time-harmonic motions of the linearly elastic isotropic heat-conducting solids are governed by the system of partial differential equations (PDEs), which can be written in the form

$$
\begin{gathered}
\left(\lambda_{T}+2 \mu_{T}\right) \nabla(\nabla \cdot \boldsymbol{u})-\mu_{T} \nabla \times \nabla \times \mathbf{u}-\gamma \nabla \theta=-\rho \omega^{2} \mathbf{u}, \\
\kappa \nabla^{2} \theta=-\mathrm{i} \omega\left[\rho C \theta+\gamma T_{0}(\nabla \cdot \mathbf{u})\right],
\end{gathered}
$$

see e.g. $[2,3]$. Here $\boldsymbol{u}$ is the displacement vector, $\theta$ the scalar field measuring the local absolute deviation from the ambient temperature $T_{0}, \omega$ the angular frequency, $\nabla$ the gradient operator, $\rho$ the material density, $\kappa$ the thermal conductivity and $C$ the specific heat at constant strain. The coupling parameter $\gamma$ is defined as

$$
\gamma=a\left(3 \lambda_{T}+2 \mu_{T}\right) \equiv \frac{2 a \mu_{T}\left(1+v_{T}\right)}{1-2 v_{T}},
$$

\footnotetext{
${ }^{1}$ Email: aleksey.pichugin@brunel.ac.uk
} 
TABLE 1. Typical values of material parameters for several metallic materials, as given in [10].

\begin{tabular}{l|cccccccc}
\hline & $\begin{array}{c}\rho, \\
\mathbf{k g} / \mathbf{m}^{3}\end{array}$ & $\begin{array}{c}\mu, \\
\mathbf{G P a}\end{array}$ & $v$ & $\begin{array}{c}a, \mathbf{K} \\
1 / \mathbf{K}\end{array}$ & $\begin{array}{c}\kappa /(\mathbf{m} \mathbf{K}) \\
\mathbf{W}\end{array}$ & $\begin{array}{c}C,(\mathbf{k g ~ K}) \\
\mathbf{J}\end{array}$ & $\boldsymbol{\varepsilon}$ & $\begin{array}{c}\alpha h, \\
\mathbf{m}\end{array}$ \\
\hline Zinc (Zn) & 7100 & 38.6 & 0.249 & $31.2 \times 10^{-6}$ & 112.2 & 389.8 & $3.33 \times 10^{-2}$ & $10 \times 10^{-9}$ \\
Lead (Pb) & 11340 & 4.9 & 0.42 & $29.1 \times 10^{-6}$ & 17.6 & 129 & $3.68 \times 10^{-2}$ & $6.77 \times 10^{-9}$ \\
Aluminium (Al) & 2700 & 25.7 & 0.33 & $24 \times 10^{-6}$ & 210 & 900 & $2.84 \times 10^{-2}$ & $14.1 \times 10^{-9}$ \\
Silver (Ag) & 10500 & 27.7 & 0.37 & $19.6 \times 10^{-6}$ & 419 & 234 & $2.97 \times 10^{-2}$ & $47.4 \times 10^{-9}$ \\
Copper (Cu) & 8960 & 41 & 0.343 & $16.4 \times 10^{-6}$ & 385 & 385 & $1.67 \times 10^{-2}$ & $25.6 \times 10^{-9}$ \\
Iron (Fe) & 7900 & 76 & 0.291 & $12.2 \times 10^{-6}$ & 76.2 & 440 & $1.1 \times 10^{-2}$ & $3.83 \times 10^{-9}$ \\
Titanium (Ti) & 4500 & 43.3 & 0.34 & $8.9 \times 10^{-6}$ & 17 & 528 & $0.743 \times 10^{-2}$ & $1.13 \times 10^{-9}$ \\
Tungsten (W) & 19300 & 135 & 0.28 & $4.4 \times 10^{-6}$ & 163.3 & 134 & $0.313 \times 10^{-2}$ & $13.2 \times 10^{-9}$ \\
Silicon (Si) & 2490 & 43.9 & 0.28 & $2.5 \times 10^{-6}$ & 84 & 670 & $0.051 \times 10^{-2}$ & $6.64 \times 10^{-9}$ \\
\hline
\end{tabular}

where $a$ is the coefficient of linear expansion and the standard Lamé modulae $\lambda_{T}$ and $\mu_{T}$, as well as the Poisson ratio $v_{T}$, are isothermal elastic constants measured at $T_{0}$.

The relative complexity of system (1)-(2) has motivated researchers to develop various schemes for the approximate treatment of initial and boundary value problems of the thermoelasticity. Traditional approximations rely upon discarding the inertial term $\rho \omega^{2} \mathbf{u}$ in the equation of motion (1) (the quasi-static approximation) and/or the coupling term $\gamma T_{0}(\nabla \cdot \boldsymbol{u})$ in the diffusion equation (2) (the approximation of the theory of thermal stresses), see $[4,5]$ and more recent discussion in [6]. Several other approximate methods were proposed for solving initial value problems, see e.g. [7, 8]. Unfortunately, all of these approximations are either too specialised to be applicable to a wider range of problems, or result in qualitative (and quantitative) differences with predictions of the fully coupled dynamic theory.

Chadwick and Sneddon performed a detailed analysis of plane waves propagating in the thermoelastic solids. In particular, they observed that propagation of the plane waves with frequencies $\omega \ll \omega_{*}$, where

$$
\omega_{*}=\rho c_{1}^{2} C / \kappa, \quad c_{1}=\sqrt{\left(\lambda_{T}+2 \mu_{T}\right) / \rho},
$$

is predominantly adiabatic, see [1]. The frequency $\omega_{*}$ for a typical metal is situated in the GHz range. The implication is that the majority of practical ultrasonic devices operate in the predominantly adiabatic regime. The characteristic unit of length $\ell=\kappa /\left(\rho c_{1} C\right)$ underlying definition (4) is proportional to the mean free path of thermal phonons, see [9]. A more practical definition of the unit frequency for mechanical oscillators should be based on the typical wavelength of interest $h \gg \ell$, that is usually commensurate with the relevant dimension of the oscillator:

$$
\bar{\omega}=\omega h / c_{2}, \text { where } c_{2}=\sqrt{\mu_{T} / \rho} .
$$

Note how we use the overbars to indicate the quantities that were non-dimensionalised. If the wavelength $h$ is selected as our unit of length, then it is convenient to choose $\gamma T_{0} /(\rho C)$ as our unit of temperature and recast problem (1)-(2) in the compact non-dimensional form

$$
\begin{gathered}
\bar{\nabla}(\bar{\nabla} \cdot \overline{\mathbf{u}})-\chi^{2} \bar{\nabla} \times \bar{\nabla} \times \overline{\mathbf{u}}-\varepsilon \bar{\nabla} \bar{\theta}=-\chi^{2} \bar{\omega}^{2} \overline{\mathbf{u}} \\
\alpha \bar{\nabla}^{2} \bar{\theta}=-\mathrm{i} \bar{\omega}[\bar{\theta}+\bar{\nabla} \cdot \overline{\mathbf{u}}],
\end{gathered}
$$

where

$$
\chi=c_{2} / c_{1}, \quad \varepsilon=\gamma^{2} \chi^{2} T_{0} /(\mu \rho C) \quad \text { and } \quad \alpha=\left[\kappa /\left(\rho c_{2} C\right)\right] / h .
$$

The coupling parameter $\varepsilon$ matches the definition given in [1]; the new "adiabaticity" parameter $\alpha=\ell /(\chi h)$ describes the degree of scale separation between the characteristic length of thermal diffusion and the typical oscillation wavelength. Representative values of these parameters are collated in Table 1. It is immediately apparent that the value of $\alpha$ remains small even for wavelengths $h$ on the scale of hundreds of nanometers. This is the reason why $\alpha$ is chosen as the natural small parameter underlying the asymptotic analysis presented in the following sections. 


\section{AN EXPANSION NEAR THE ADIABATIC LIMIT}

If parameter $\alpha$ is negligibly small and $\bar{\omega} \sim 1$, the diffusion equation (7) reduces to the standard adiabatic relationship $\bar{\theta}=-\bar{\nabla} \cdot \overline{\boldsymbol{u}}$. When it is substituted into PDEs (6), one obtains the classic elastodynamic equations

$$
\bar{\nabla}(\bar{\nabla} \cdot \overline{\mathbf{u}})-\frac{\chi^{2}}{1+\varepsilon} \bar{\nabla} \times \bar{\nabla} \times \overline{\mathbf{u}}=-\frac{\chi^{2}}{1+\varepsilon} \bar{\omega}^{2} \overline{\mathbf{u}},
$$

in which the isothermal elastic constant $\chi^{2}$ is replaced by the adiabatic one $\chi^{2} /(1+\varepsilon)$, see [3].

This approximation can be refined by using system (6)-(7) and seeking

$$
\overline{\mathbf{u}}=\overline{\mathbf{u}}_{0}+\alpha \overline{\mathbf{u}}_{1}+\ldots, \quad \overline{\boldsymbol{\theta}}=\bar{\theta}_{0}+\alpha \bar{\theta}_{1}+\ldots .
$$

Clearly, the leading order displacement $\overline{\mathbf{u}}_{0}$ satisfies (9), while the leading order temperature increment remains adiabatic: $\bar{\theta}_{0}=-\bar{\nabla} \cdot \overline{\mathbf{u}}_{0}$. The departure from the adiabaticity is captured at the next order, where

$$
\bar{\theta}_{1}=-\bar{\nabla} \cdot \overline{\mathbf{u}}_{1}+\frac{\mathrm{i} \chi^{2} \bar{\omega}}{1+\varepsilon} \bar{\nabla} \cdot \overline{\mathbf{u}}_{0},
$$

so that

$$
\bar{\nabla}\left(\bar{\nabla} \cdot \overline{\mathbf{u}}_{1}\right)-\frac{\chi^{2}}{1+\varepsilon} \bar{\nabla} \times \bar{\nabla} \times \overline{\mathbf{u}}_{1}-\frac{\mathrm{i} \varepsilon \chi^{4} \bar{\omega}}{(1+\varepsilon)^{3}}\left[\bar{\nabla} \times \bar{\nabla} \times \overline{\mathbf{u}}_{0}-\bar{\omega}^{2} \overline{\mathbf{u}}_{0}\right]=-\frac{\chi^{2}}{1+\varepsilon} \bar{\omega}^{2} \overline{\mathbf{u}}_{1} .
$$

Expansions (10) can now be used to combine equations (9) and (12) into a simple system of PDEs given by

$$
\bar{\nabla}(\bar{\nabla} \cdot \overline{\mathbf{u}})-\chi_{*}^{2} \bar{\nabla} \times \bar{\nabla} \times \overline{\mathbf{u}}=-\chi_{*}^{2} \bar{\omega}^{2} \overline{\mathbf{u}}+O\left(\alpha^{2}\right), \quad \text { where } \quad \chi_{*}^{2}=\frac{\chi^{2}}{1+\varepsilon}+\frac{\mathrm{i} \alpha \varepsilon \chi^{4} \bar{\omega}}{(1+\varepsilon)^{3}} .
$$

System (13), featuring the frequency-dependent elastic module $\chi_{*}$, is formally equivalent to the classic Navier equations for the time-harmonic motion of isotropic elastic media. This equivalence should be understood in the same spirit as the viscoelastic correspondence principle, based on the formal equivalence between time-harmonic solutions of elastic and viscoelastic boundary value problems, see [11]. In fact, our expression for $\chi_{*}^{2}$ is equivalent to the elastic module obtained in the time-harmonic case for the Voight model of viscoelastic solid.

"Quasi-adiabatic" (or "low-loss") approximations equivalent to (13) are described in the literature; they are discussed at some length in [6,12], and the propagation of plane waves in an equivalent low-loss media is studied in [13]. Unfortunately, the pleasant simplicity of model (13) comes at a price: it assumes a specific dependence between the thermal and displacement fields, which can be recovered from equations (10), (11) and (13) as

$$
\bar{\theta}=-\left[1-\mathrm{i} \alpha \chi_{*}^{2} \bar{\omega}\right] \bar{\nabla} \cdot \overline{\mathbf{u}}+O\left(\alpha^{2}\right) .
$$

As a consequence, the described quasi-adiabatic approximation is, in general, not capable of satisfying the temperature boundary conditions naturally occurring in thermoelastic boundary value problems. This situation is common in the asymptotic analysis of PDEs; it signals of the presence of a thermoelastic boundary layer.

\section{THE THERMOELASTIC BOUNDARY LAYER}

We begin our analysis by postulating the expected form of the solution for our problem:

$$
\overline{\mathbf{u}}=\overline{\boldsymbol{u}}^{i}+\overline{\boldsymbol{u}}^{b l}, \quad \bar{\theta}=\bar{\theta}^{i}+\bar{\theta}^{b l} .
$$

The "inner" solution $\left(\overline{\boldsymbol{u}}^{i}, \bar{\theta}^{i}\right)$ is required to satisfy the quasi-adiabatic equations (13) and (14). The "boundary layer" terms $\overline{\boldsymbol{u}}^{b l}$ and $\bar{\theta}^{b l}$ represent rapidly varying fields localised in the vicinity of boundaries, which enable one to satisfy the correct boundary conditions of thermoelasticity.

Suppose that the boundary of interest is aligned with one of the coordinate surfaces within the chosen coordinate system. This means that we can rescale the space to "zoom" into the vicinity of the boundary. The scaling transformation $\xi=\boldsymbol{x} / \sqrt{\alpha}$ results in $\bar{\nabla}=\bar{\nabla} \xi / \sqrt{\alpha}$, hence transforming governing equations (6)-(7) into

$$
\begin{gathered}
\bar{\nabla}_{\xi}\left(\bar{\nabla}_{\xi} \cdot \overline{\boldsymbol{u}}^{b l}\right)-\chi^{2} \bar{\nabla}_{\xi} \times \bar{\nabla}_{\xi} \times \overline{\boldsymbol{u}}^{b l}-\sqrt{\alpha} \varepsilon \bar{\nabla}_{\xi} \bar{\theta}^{b l}=-\alpha \chi^{2} \bar{\omega}^{2} \overline{\boldsymbol{u}}^{b l}, \\
\sqrt{\alpha}\left[\bar{\nabla}_{\xi}^{2} \bar{\theta}^{b l}+\mathrm{i} \bar{\omega} \bar{\theta}^{b l}\right]=-\mathrm{i} \bar{\omega} \bar{\nabla}_{\xi} \cdot \overline{\mathbf{u}}^{b l} .
\end{gathered}
$$


The boundary layer solution for equations (16)-(17) can be constructed by seeking expansions of the form

$$
\overline{\mathbf{u}}^{b l}=\sqrt{\alpha}\left(\overline{\mathbf{u}}_{0}^{b l}+\alpha \overline{\mathbf{u}}_{1}^{b l}+\ldots\right), \quad \bar{\theta}^{b l}=\bar{\theta}_{0}^{b l}+\alpha \bar{\theta}_{1}^{b l}+\ldots,
$$

so that the leading order solution for boundary layer must satisfy the reduced thermoelastic problem

$$
\begin{gathered}
\bar{\nabla}_{\xi}\left(\bar{\nabla}_{\xi} \cdot \overline{\boldsymbol{u}}_{0}^{b l}\right)-\chi^{2} \bar{\nabla}_{\xi} \times \bar{\nabla}_{\xi} \times \overline{\mathbf{u}}_{0}^{b l}-\varepsilon \bar{\nabla}_{\xi} \bar{\theta}_{0}^{b l}=O(\alpha), \\
\bar{\nabla}_{\xi}^{2} \bar{\theta}_{0}^{b l}+\mathrm{i} \bar{\omega} \bar{\theta}_{0}^{b l}=-\mathrm{i} \bar{\omega} \bar{\nabla}_{\xi} \cdot \overline{\mathbf{u}}_{0}^{b l} .
\end{gathered}
$$

The quasi-static nature of equations (19)-(20) represents a simplification of the original problem. A uniform dilation of space implied by our zooming transformation $\xi=\mathbf{x} / \sqrt{\alpha}$ did not take into account a possible spatial non-uniformity of the boundary layer, which means that the dimensionality of system (19)-(20) can also be reduced in some cases. Even further simplification is possible for boundary layers dominated by dilatation, such that $\bar{\nabla}_{\xi} \times \overline{\boldsymbol{u}}_{0}^{b l} \ll 1$. In this case equation (19) can be integrated to find that $\bar{\nabla}_{\xi} \cdot \overline{\mathbf{u}}_{0}^{b l}=\varepsilon \bar{\theta}_{0}^{b l}+O(\alpha)$, with remaining equation (20) furnishing the explicit representation for the thermal boundary layer

$$
\bar{\nabla}_{\xi}^{2} \bar{\theta}_{0}^{b l}+\mathrm{i} \bar{\omega}(1+\varepsilon) \bar{\theta}_{0}^{b l}=O(\alpha) .
$$

The "boundary layer dominated by dilatation" may appear to be a somewhat artificial construct, rarely achievable due to the complicated nature of the boundary conditions of elasticity. Nevertheless, such situation is indeed possible, for instance, for purely one-dimensional longitudinal motions. In this important case the leading order boundary layer solution is given simply by $\bar{\theta}_{0}^{b l}=\bar{\Theta}_{0}^{b l} \exp [\mp(1-\mathrm{i}) \sqrt{\omega(1+\varepsilon) /(2 \alpha)} x]$.

Let us consider a $1 \mathrm{D}$ thermoelastic problem for $x \geqslant 0$ with the fixed boundary $\left.\bar{u}\right|_{x=0}=\bar{d}$ kept at a fixed temperature: $\left.\bar{\theta}\right|_{x=0}=0$. It follows from representation (15), adiabatic condition (14) and boundary layer equation (21) that

$$
\left.\bar{\theta}\right|_{x=0}=\left.\bar{\theta}^{i}\right|_{x=0}+\left.\bar{\theta}^{b l}\right|_{x=0}=-\left.\bar{u}_{, x}^{i}\right|_{x=0}+\left.\bar{\theta}_{0}^{b l}\right|_{x=0}+O(\alpha)=0,
$$

from which it is clear that $\left.\left.\bar{\theta}_{0}^{b l}\right|_{x=0} \sim \bar{u}_{, x}^{i}\right|_{x=0}$ (note that the comma subscripts signify differentiation with respect to the indicated parameter). We also know that $\sqrt{\alpha} \bar{u}_{0, x}^{b l}=\varepsilon \bar{\theta}_{0}^{b l}+O(\alpha)$, which can be integrated to obtain $\left.\bar{u}_{0}^{b l}\right|_{x=0}=$ $-\left.\varepsilon \bar{\theta}_{0}^{b l}\right|_{x=0} \cdot(1+\mathrm{i}) / \sqrt{2 \omega(1+\varepsilon)}+O(\sqrt{\alpha})$. This means that the effective boundary condition should be given by

$$
\left.\bar{u}\right|_{x=0}=\left[\bar{u}^{i}+\bar{u}^{b l}\right]_{x=0}=\left[\bar{u}^{i}+\sqrt{\alpha} \bar{u}_{0}^{b l}+O\left(\alpha^{3 / 2}\right)\right]_{x=0}=\left[\bar{u}^{i}-\frac{(1+\mathrm{i}) \varepsilon \sqrt{\alpha}}{\sqrt{2 \bar{\omega}(1+\varepsilon)}} \bar{u}_{, x}^{i}+O(\alpha)\right]_{x=0}=\bar{d} .
$$

It is worth noting that the $O(\sqrt{\alpha})$ amplitude adjustment evident from (23) cannot in principle be reproduced by quasiadiabatic approximation (13), even if higher order corrections are considered. This situation is reminiscent of the difficulties one is facing when trying to refine theories of thin plates: the superficial accuracy of the inner expansions is invariably capped by the error in reproducing the correct (non-asymptotic) boundary conditions, see [14].

\section{ACKNOWLEDGMENTS}

The author gratefully acknowledges support by the "BRIEF" research award (Brunel University).

\section{REFERENCES}

1. P. Chadwick, and I. N. Sneddon, Journal of the Mechanics and Physics of Solids 6, 223-230 (1958).

2. J. D. Achenbach, Wave Propagation in Elastic Solids, North-Holland Publishing Co., Amsterdam, 1973.

3. W. Nowacki, Dynamic Problems of Thermoelasticity, Noordhoff International Publishing, Leyden, 1975.

4. P. H. Francis, Journal of Sound and Vibration 21, 181-192 (1972).

5. W. A. Day, Archive for Rational Mechanics and Analysis 77, 387-396 (1981).

6. Z. Płochocki, and A. Mioduchowski, Transactions of the Canadian Society for Mechanical Engineering 27, 31-46 (2003).

7. R. B. Hetnarski, Bulletin de l'Académie Polonaise des Sciences: Série des Sciences Techniques 12, $49-57$ (1964).

8. J. D. Achenbach, Journal of the Acoustical Society of America 36, 10-18 (1964).

9. R. E. Peierls, Quantum Theory of Solids, Clarendon Press, Oxford, 2001. 
10. R. B. Ross, Metallic Materials Specification Handbook, E. \& F. N. Spon, London, 1980, 3rd edn.

11. R. M. Christensen, Theory of Viscoelasticity: An Introduction, Academic Press, New York, 1982, 2nd edn.

12. L. D. Landau, and E. M. Lifshitz, Course of Theoretical Physics, vol. 7: Theory of Elasticity, Pergamon Press, Oxford, 1975, 2nd edn.

13. A. L. Shuvalov, and N. H. Scott, Acta Mechanica 140, 1-15 (2000).

14. J. D. Kaplunov, L. Yu. Kossovich, and E. V. Nolde, Dynamics of Thin Walled Elastic Bodies, Academic Press, New York, 1998. 